\title{
Cardiovascular and smooth muscle pharmacology in the next decade
}

\author{
Jeffrey Atkinson* \\ Nancy University, Nancy, France \\ *Correspondence: Jeffrey.atkinson@pharma.uhp-nancy.fr
}

In 2008, half of the 15 best-selling cardiovascular drugs (those with sales of over $\$ 2$ billion), the sartans, were developed over 30 years ago. A lipid-lowering drug developed over 40 years ago (fenofibrate) also made it into the top fifteen. Rare are those developed more recently. One example is ezetimibe developed in the 1990s. Drugs acting on cholesterol levels such as fenofibrate, ezetimibe and the statins represent over a quarter of the top fifteen. There are many reasons behind these figures - the duration of the drug development process from synthesis to marketing, the economics of the pharmaceutical industry that in some markets make it more financially rewarding to copy the others than to innovate, etc. Albeit, looking at these figures, a pessimist would be tempted to say that cardiovascular pharmacologists have shown a certain lack of imagination - an optimist would say that there is a large number of potential targets out there just waiting to be discovered and developed.

Frontiers in cardiovascular and smooth muscle pharmacology will focus on such potential targets and concepts, as well as on the new technology and methodology required to investigate them.

Classical drug research and development was based on the identification of selective membrane targets such as receptors or ion channels that promote a specific reaction to a given drug in the midst of the chemical cacophony of the receptor or ion channel biophase. The invention by Sir James Black of propranolol, the betaadrenoreceptor antagonist, in the 1960s, was based on the pioneering work done by himself and others on the development of specific ligands for specific adrenoreceptor types. This drug revolutionized the management of angina pectoris and is one of the most important contributions to clinical pharmacology of the 20th century. In the 1960s Black was also cogitating on other applications of receptor pharmacology.
He thought that the histamine antagonists available at that time were analogous to alpha-adrenoreceptor antagonists and that the equivalent of propranolol was needed to block histamine-stimulated acid secretion in the stomach. This lead in the 1970s to the $\mathrm{H}_{2}$-receptor antagonist programme and cimetidine. Such an approach is still highly relevant today and investigation into the roles of 5-HT2Band 5-HT7 receptors as well as 5-HT3-receptor subunits in the gastrointestinal pharmacology and physiology of 5-HT is of prime importance. Given that there are some $800 \mathrm{G}$-protein coupled receptors, this classical membrane receptor approach still has a bright future.

Another concept that dates from the 1960 s is that of receptor desensitization. At that time the idea started to emerge that agonists may not only stimulate receptors but that they can also desensitize them. Interesting work is now being done on the desensitization of G-protein coupled receptors through phosphorylation and the recruitment of arrestins. This process appears to be uniquely related to the cell type in which the receptor is expressed, thus opening up an avenue for research into agents that selectively modulate receptor desensitization in one type of cell but not in another.

Another approach to the development of therapeutic agents is to interfere with the signal before it reaches the receptor or whatever its target may be. A novel way to do this is to use "immunodrugs" that are therapeutic vaccines intended for the treatment and prevention of common chronic diseases. In hypertension current vaccination approaches target angiotensin I and II. It is interesting that the vaccine appears to be very effective in the early morning, a critical time of day when adverse cardiovascular events occur frequently.

Pharmacology went beyond the receptor many years ago. Over the years there has been growing interest in the cellular mechanisms of signal transduction and amplification behind receptors and the clinical applications of these mechanisms. For example, work on the erythropoietin intracellular signalling cascade, has shown that this agent can limit infarct size following myocardial ischemia by activating survival kinases of the RISK pathway including PI3K/Akt and ERK1/2during reperfusion.

The development of new physiological concepts will also furnish new ideas for cardiovascular and smooth muscle pharmacology. It was recently showed that in the elderly an increase in pulse blood pressure arising from increased large artery stiffness can have detrimental effects on cardiovascular structure and function. This has opened up an exciting domain of research into agents capable of directly modifying wall stiffness (rather than arterial diameter as was classically the case). Such agents interfere, for example, with physicochemical processes such as glycation and calcification of long-lived scleroproteins (collagen and elastin). The research and development of these agents will require novel pharmacological paradigms.

Modulation of arterial diameter has not, however, delivered all of its secrets. Another interesting new concept is that of the resetting of myogenic tone. The arteriolar myogenic response is defined as vasoconstriction to an increase in intra-luminal pressure and dilation to a reduction in pressure. This response assures the control of microvascular diameter and hence tissue blood flow. Although progress has been made in research on the sensory apparatus and cellular mechanisms involved, other new areas like the pharmacological modulation of the interactions between matrix proteins and the cell membrane are opening up.

Developments in drug formulation and delivery systems will also be important in future therapy. Thus, for example, non-invasive delivery systems represent a novel approach for gene, cell and drug delivery to damaged tissue. An example of 
this is ultrasound-mediated micro-bubble destruction that has been applied in the targeted delivery of genes, drugs and stem cells to the myocardium following infarction.

Advances in modelling are also proving useful to pharmacological drug development. Pharmacokinetics promises to bring new facets to drug research. The use of pharmacokinetic-pharmacodynamic modelling will lead to better understanding of drug efficacy and safety by predicting efficacy and safety in humans on the basis of bioassay data and data from in vivo safety pharmacology. Other modelling approaches involve the application of systems biology to pharmacology and drug development. Systems biology describes complex biological systems with the aim of developing predictive models of disease. Genomics, proteinomics and metabolomics have accelerated understanding in disease models and mathematical simulations integrating knowledge of organ and system-level responses can help identify drug targets in disease.

Modern day cardiovascular and smooth muscle pharmacology represents a fascinating, multi-faceted, fast developing science. Frontiers in cardiovascular and smooth muscle pharmacology hopes to bring you the up-to-date developments in this science.

Received: 27 January 2010; accepted: 11 February 2010; published online: 19 May 2010

Citation: Atkinson J (2010) Cardiovascular and smooth muscle pharmacology in the next decade. Front. Pharmacol. 1:1. doi: 10.3389/fphar.2010.00001

This article was submitted to Frontiers in Cardiovascular and Smooth Muscle Pharmacology, a specialty of Frontiers in Pharmacology.

Copyright $(\odot 2010$ Atkinson. This is an open-access article subject to an exclusive license agreement between the authors and the Frontiers Research Foundation, which permits unrestricted use, distribution, and reproduction in any medium provided the original authors and source are credited. 